\section{Personalized medicine gets personal}

\section{By Tracey Baas, Senior Editor}

Epithelial cancers are the most common malignancies, but immunotherapy development has been stymied because of the difficulty in identifying targets that are not expressed on normal tissues. A National Cancer Institute team has now used a next-generation sequencing approach to identify such rare mutations in one patient and determine which, if any, are recognized by the immune system. The team then developed an autologous cell therapy against one of the mutations that led to cancer regression and disease stabilization. ${ }^{1}$

The researchers now need to show that the personalized method can provide similar results for unique mutations in other patients and that the approach can further be generalized to treat large numbers of patients.

"It isn't known at present how frequently actionable, i.e., immunogenic, mutations are to be found commonly in epithelial cancers," said Michel Sadelain, director of the Memorial Sloan-Kettering Cancer Center's Center for Cell Engineering and cofounding scientist of Juno

\section{“It isn't known at present} how frequently actionable, i.e., immunogenic, mutations are to be found commonly in epithelial cancers. Methods to rapidly identify the relevant immunogenic mutations are direly needed."

- Michel Sadelain, Memorial Sloan-Kettering Cancer Center
(ERBB2IP) antigen containing an E805G mutation via the T cell receptor (TCR) V $\beta 22^{+}$.

The patient received adoptive cell therapy that used about 42 billion expanded TILs and included more than 10 billion of the CD4 ${ }^{+}$, ERBB2IP ${ }^{\mathrm{E} 805 \mathrm{G}}$-reactive $\mathrm{T}$ cells. The time from whole-exome sequencing to first cell therapy dosing was about six weeks.

The treatment resulted in up to $29 \%$ tumor regression in the patient's lung and liver lesions. Disease stabilization lasted for about 13 months, after which disease progressed in the lungs but not the liver.

Following relapse, the patient was treated with a $\mathrm{T}$ cell product still regressing at 7 months.

The results were published in Science.

\section{Moving to prime time}

Rosenberg cautioned that much more work is needed to turn the method into a therapy suitable for widespread use.

"Although our one-patient study demonstrates that low-frequency mutation-specific reactive $\mathrm{T}$ cells can be found and expanded from patient TILs to generate a $\mathrm{T}$ cell product to treat epithelial cancers, this strategy is not yet ready for prime time," said Rosenberg. "We need more than an $\mathrm{N}$ of one."

Michael Gladstone, an associate in the life sciences group at Atlas Venture, said that the strategy might be more practical if it were redirected to target frequent cancer mutations rather than patient-specific mutations.

"It would be helpful to see how readily and robustly mutation-specific tumor antigens Therapeutics Inc. "Methods to rapidly identify the relevant immunogenic mutations are direly needed.'

The NCI team, led by Steven Rosenberg, set out to identify mutationspecific tumor-infiltrating lymphocytes (TILs) in epithelial cancers and characterize the TIL responses against the tumors.

Rosenberg is chief of surgery at the NCI and a professor of surgery at the Uniformed Services University of the Health Sciences and George Washington University School of Medicine and Health Sciences.

His group focused on one patient with widely metastatic cholangiocarcinoma-a rapid and incurable form of GI cancer that arises from mutated epithelial cells in the bile ducts.

The team obtained lung metastatic tissue to use for whole-exome sequencing and identified 26 mutations.

To determine if the patient's TILs could recognize and react to any of these mutations, the team transfected RNAs representing the 26 mutations into ex vivo antigen-presenting cells from the patient and cocultured them with the patient's TILs.

The researchers identified one set of $\mathrm{CD} 4^{+} \mathrm{T}$ cells that specifically recognized dendritic cells presenting an ErbB2 interacting protein can be identified from exome sequencing in different patients and different tumor types," said Gladstone. "It is possible that identifying potent, mutation-specific $\mathrm{T}$ cell populations will be easier in some patients than others, and it is not clear how broadly deployable this type of personalized therapy can be made to other cancer centers."

Rosenberg agreed. "We have done some additional preliminary work with three other patients who have epithelial-derived colorectal cancers," he told SciBX. "We used whole-exome sequencing to identify cancer-associated mutations in their metastatic tissues. When we used the cocultures of patient TILs and RNA-transfected APCs as described in our study, we found patient-specific $\mathrm{T}$ cells that were mutation reactive in two of the three patients."

"Many mutations may not be targeted by a T cell population because the immune system might not process the antigen in a way that would be recognized by T cells," continued Rosenberg. "Our method provides the opportunity to identify endogenous T cell populations that can be used for cell therapy but depends on the existence of an endogenous, patient-specific, mutation-reactive T cell population." 


\section{"Although our one-patient study demonstrates that low- frequency mutation- specific, reactive $T$ cells can be found and expanded from patient TILs to generate a $\mathrm{T}$ cell product to treat epithelial cancers, this strategy is not yet ready for prime time. We need more than an $\mathrm{N}$ of one." \\ -Steven Rosenberg, National Cancer Institute}

Gladstone said that because of the complexity of the NCI's cell therapy procedure, it would likely be reserved for the most severe and treatment-refractory patents. He did say it would be more scalable if patients were instead screened for a panel of relatively frequent mutations.

"In this way, cancer centers could establish master cell banks of relevant antigen-presenting cells for TIL expansion or could generate engineered TCRs or CARs [chimeric antigen receptors] that can be ectopically expressed in autologous or allogeneic
T cells via viral transduction," said Gladstone.

The NCI has filed for a patent application covering the findings.

Baas, T. SciBX 7(21); doi:10.1038/scibx.2014.602

Published online May 29, 2014

\section{REFERENCES}

1. Tran, E. et al. Science; published online May 9, 2014; doi:10.1126/science.1251102

Contact: Steven A. Rosenberg, National Cancer Institute, Bethesda, Md.

e-mail: sar@nih.gov

COMPANIES AND INSTITUTIONS MENTIONED

Atlas Venture, Cambridge, Mass.

George Washington University School of Medicine and Health Sciences, Washington, D.C.

Juno Therapeutics Inc., Seattle, Wash.

Memorial Sloan-Kettering Cancer Center, New York, N.Y.

National Cancer Institute, Bethesda, Md.

Uniformed Services University of the Health Sciences, Bethesda, Md. 